\title{
Unique detection of organic vapors below their auto-ignition temperature using III-V Nitride based triangular microcantilever heater
}

Ifat Jahangir, * Ehtesham Bin Quddus, and Goutam Koley

Ifat Jahangir

Department of Electrical Engineering, University of South Carolina, Columbia, SC 29208, USA

Ehtesham Bin Quddus

Intel Corporation, Portland, Oregon, 97208, USA

Goutam Koley

Department of Electrical and Computer Engineering, Clemson University, Clemson, SC 29634, USA

*Corresponding author. E-mail: ifat00@gmail.com, Phone: +1-803-445-8006

Mailing address: 301 S Main St, Room no 3A80, Columbia, SC 29208, USA

Keywords: Microcantilever, $\mathrm{AlGaN} / \mathrm{GaN}$ heterostructure, polarization, heater, organic vapor

We report on the unique detection of dilute volatile organic compounds below their autoignition temperature using a novel $\mathrm{AlGaN} / \mathrm{GaN}$ heterostructure based triangular microcantilever heater. With a low input power of $12 \mathrm{~mW}$, the microcantilever heater was found to reach a maximum temperature of $330{ }^{\circ} \mathrm{C}$ at the tip, which was verified through infrared microscopy and Raman spectroscopy. Unique threshold voltages were observed for various organic analytes with different functional groups, beyond which the heater current started to increase sharply in presence of analytes, which correlated strongly with their latent

31 heat of evaporation. Dilute vapors with concentrations as low as 50 parts per million (ppm) 32 could be detected selectively with a noise limited resolution down to $5 \mathrm{ppm}$. On the other

33 hand, the magnitude of change of current for a fixed applied voltage was found to be 34 dependent on the molecular dipole moment of the analytes, which can likely be attributed to 35 the strong surface polarization of $\mathrm{AlGaN}$. A simple circuit model has been proposed to 36 explain the observations. Heat transfer and Joule heating simulations were performed using 
1 finite element method to model the electro-thermal characteristics of the microcantilever

2 heater, which were in good agreement with the experimental observations.

3

4 1. Introduction

5 Detection of volatile organic compounds (VOCs), which are widely used in industrial 6 processes and household products, is very important due to significant health hazards 7 associated with them. ${ }^{[1]}$ VOCs are commonly detected using photo-ionization detectors 8 (PIDs), ${ }^{[2]}$ suspended hot bead pellistors, ${ }^{[3]}$ or heated metal oxide semiconductor 9 functionalization layers. ${ }^{[4]-[7]}$ The detection methodology using PIDs is based on high-energy 10 photon (typically $>10.5 \mathrm{eV}$ ) induced ion generation, while that using hot bead pellistors takes 11 advantage of the exothermic reaction (from auto-ignition of VOCs) to produce a change in 12 resistance. Heated metal oxide (i.e. $\mathrm{TiO}_{2}$ or $\mathrm{SnO}_{2}$ ) based sensing also relies upon a change is 13 resistance, but at a temperature below the auto-ignition temperature of the VOCs. However, 14 all the above techniques suffer from the problem of high power requirement as well as poor 15 selectivity among VOCs, which is often important for proper identification of the source of a problem. Although the last method requires somewhat lower operational power, it involves 17 complicated functionalization process with metal oxides.

Microcantilevers offer excellent avenues for molecular sensing that arises out of their high sensitivity to various physical parameter changes induced by the analyte molecules. ${ }^{[8]-[15]}$ 21 Microcantilever heaters, which are extremely sensitive to changes in thermal parameters, ${ }^{[16]-}$ $22{ }^{[21]}$ have been widely utilized for calorimetry, ${ }^{[16]}$ thermal nanotopography ${ }^{[17]}$ and thermal 23 conductivity measurements. ${ }^{[18]}$ Due to the small area of the microcantilever that needs to be 24 heated (i.e. the tip of a triangular microcantilever), they also offer the possibility of reduced 25 power consumption for high temperature operation. However, achieving repeatable and 26 reliable functionalization of a microcantilever, especially over a small area, is a challenge that 
1 has thwarted practical applications of microcantilever based sensors. On the other hand,

2 unfunctionalized microcantilevers (typically made of $\mathrm{Si}$ ) are not particularly sensitive toward

3 a specific analyte, and are generally accepted to be incapable of performing selective

4 detection. Thus, only a handful of studies utilizing uncoated microcantilevers to perform

5 unique molecular detection have been reported so far. ${ }^{[13],[22],[23]}$ In these studies, detection is

6 generally based on changes in physical properties of the media surrounding the cantilever (i.e.

7 viscosity, ${ }^{[23]}$ thermal conductivity, ${ }^{[22]}$ or the analyte (i.e. deflagration temperature ${ }^{[22]}$ ).

8 However, these techniques are applicable only to a few specific analytes, and selective

9 detection still remains a major challenge, especially when the analytes are diluted (or present

10 in minute quantities) or have similar physical properties i.e. VOCs.

III-Nitride heterojunction (especially $\mathrm{AlGaN} / \mathrm{GaN}$ ) based microcantilevers offers a 13 unique opportunity for realizing these microscale heaters, taking advantage of presence of 14 high carrier (electron) density in close proximity to the surface, ${ }^{[24]}$ which allows for highly 15 efficient surface heating. In addition, strong spontaneous polarization of III-Nitride surfaces allows these heaters to interact better with VOCs, which are typically strongly polar in nature.

17 Finally, AlGaN/GaN heterojuntion based heaters are capable of operating at high temperature and harsh environment due to chemical inertness and wide bandgap of III-Nitrides. With commercial availability of high quality III-Nitride heterojunciton epilayers on $\mathrm{Si}$, the fabrication of these heaters are also quite straightforward. Although, III-Nitride based 21 microcantilevers have been demonstrated earlier, ${ }^{[11],[25],[26]}$ there is no report so far on triangular microcantilever heaters and their sensing applications.

In this article, we present a novel $\mathrm{AlGaN} / \mathrm{GaN}$ heterostructure based triangular microcantilever heater $(\mathrm{TMH})$, demonstrated for the first time, which has been utilized to 26 perform selective detection of a number of VOCs belonging to various functional groups, 
1 including alcohols, ethers, ketones, alkanes and aromatic compounds. The detection occurs

2 below the auto-ignition temperature of the analytes and the responses have been found to

3 strongly correlate with their latent heat of evaporation and dipole moment. A simple model

4 has been presented to explain the observed responses, which are also correlated with results

5 from numerical simulations.

\section{Materials and Methods}

8 The triangular cantilevers used in this work were fabricated using $\mathrm{AlGaN} / \mathrm{GaN}$

9 heterostructure epitaxial layers grown on a $625 \mu \mathrm{m}$ thick (111) Si substrate. The microcantilevers were designed to have tapered arms (shown in the inset of Figure 1a) to

11 maximize the temperature rise at their tip under an applied bias. We started our process with

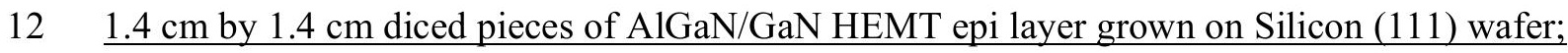

13 the details of the wafer can be found in Section I of the supporting information. At first,

$14 \underline{\mathrm{Cl}}_{2} / \mathrm{BCl}_{2} \underline{\text { Plasma was used to isolate the cantilever mesa, followed by another deeper etch to }}$

15 define the $\mathrm{GaN}$ outline. Then $\mathrm{Ti} / \mathrm{Al} / \mathrm{Ti} / \mathrm{Au}$ metal stack was deposited using an electron beam

16 evaporator, followed by rapid thermal annealing to make good ohmic contact. After that the

$17 \mathrm{Si}$ at the bottom of the pocket was etched from the backside of the sample using Bosch

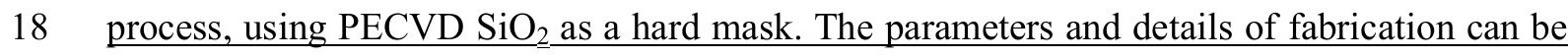

19 found in Section I of the supporting information. The total thickness of the cantilever was 2.3

$20 \mu \mathrm{m}$; base width of each arm of the cantilever was $25 \mu \mathrm{m}$, which reduced to $3 \mu \mathrm{m}$ at the tip.

21 The arms were $100 \mu \mathrm{m}$ apart at the base of the cantilever. Details of the wafer structure and

22 the fabrication process are given in Section I of supporting information and also in ${ }^{[25],[26]}$.

24 The experimental setup used for sensing volatile organic compounds involved a test 25 chamber fitted with an inlet and an outlet. Two mass flow controllers (MFC) were used to 26 control the composition of the VOC vapor (produced by passing ultra-high purity (UHP) $\mathrm{N}_{2}$ 
1 through a bubbler at room temperature), which was diluted with UHP $\mathrm{N}_{2}$. The outlet of the

2 chamber was connected to a dry pump to quickly remove the analyte from the test chamber.

3 The electrical and sensing characterizations were performed using an Agilent B2902 source

4 measuring unit (SMU).

5

\section{3. Results and Discussion}

7 The I-V characteristics of the TMH are shown in Figure 1a where a linear (low bias) and 8 a saturation region (high bias) can be clearly identified. Because of the significant self-heating

9 of the device, current is generally observed to decrease at higher biases $(>20 \mathrm{~V})$ as tip resistance increases rapidly with rise in temperature. The SEM image of the device is shown in the inset. Figure $1 \mathrm{~b}$ shows $>300 \%$ change in resistance (from 50 to $200 \mathrm{k} \Omega$ ) as the bias

12 changes from 0 to $50 \mathrm{~V}$, with a power dissipation of $12 \mathrm{~mW}$ at $50 \mathrm{~V}$. For our sensing 13 experiments, dilute analyte vapors were flown into the test chamber for a fixed duration under 14 a constant dc bias applied to the device. The wire-bonded device mounted on a 28-pin dual 15 inline package (DIP) is shown in the inset.

The device response upon exposure to $2000 \mathrm{ppm}$ molar concentration of hexane vapor is shown in Figure 2a, where the current changes from 245.8 to $250.2 \mu \mathrm{A}$ (1.84\% change) in about $30 \mathrm{~s}$, at a device bias of $50 \mathrm{~V}$. The rise time, defined by the time taken by the current to go from $10 \%$ to $90 \%$ of the steady state value, is found to be $\sim 14 \mathrm{~s}$ (Figure 2a). The fall time

21 (defined as the time for the maximum signal to decay from $90 \%$ to $10 \%$ of its value) was 22 found to be $\sim 20 \mathrm{~s}$. Comparable rise and fall times were observed for other analytes as well. 23 For comparison, the response to $2000 \mathrm{ppm}$ molar concentration of water vapor is also shown 24 for a bias of $50 \mathrm{~V}$. Since no response is observed, it confirms the lack of interference from 25 water vapor. In Figure $\mathbf{2 b}$, the response of the device to $400 \mathrm{ppm}$ of hexane vapor is shown 26 for two consecutive cycles to demonstrate complete recovery and repeatability of the device. 
1 For each phase of exposure, a current change of $1.1 \%$ was observed with an increased rise

2 time $(25-30 \mathrm{~s})$ and fall time $(\sim 35 \mathrm{~s})$, which is expected at lower concentration. The same

3 device has been used repeatedly for the same analyte and for different analytes over several

4 months, no hysteresis was observed at any occasion. The variation in sensor response has

5 been observed to be within $\pm 2 \%$ of its average value for multiple instances of sensing

6 performed for over a year, showing excellent accuracy and repeatability. Although the I-V

7 characteristics of various devices in the same batch has slight variation $(<5 \%)$, the normalized

8 response (e.g. percentage change in current) has been found to be very uniform for all sample

9 devices, with an average deviation of $\pm 3 \%$ or less for the response of different devices under

$10 \quad$ various bias/sensing conditions.

In Figure 3, temperature profile along the length of the TMH is shown using infrared 13 (IR) thermal microscopy and Raman spectroscopy. The tapered shape of the cantilever gives 14 rise to a sharp temperature variation along the arms, especially near the tip, as observed from 15 the infrared (IR) image of the cantilever (Figure 3) recorded using a temperature calibrated Inframetrics PM280 Ultra Cam fitted with IR microscopic lenses (from Sierra Pacific

17 Innovations Corporation). The microcantilever is a two-terminal self-heating device with the conducting channel serving as the heater. The entire V-shaped arm of the cantilever acts as

19 the heater-resistor. Temperature change in the arm, especially near the tip region controls the 20 change in resistance between the two ohmic contacts at the base. To determine the impact of 21 analyte flow on the temperature profile of the cantilever arms, IR images of the cantilever 22 with and without the presence of isopropanol were recorded. In Figure 3a, the cantilever 23 (dashed line) is shown at $50 \mathrm{~V}$ bias without any analyte flow, the red region at the tip is the 24 hottest spot with a temperature of about $330^{\circ} \mathrm{C}$. Figure $3 \mathrm{~b}$ shows the cantilever image with $252000 \mathrm{ppm}$ molar concentration of isopropanol vapor under the same $50 \mathrm{~V}$ bias. Figure 3c 26 shows a line scan depicting average temperature variation along the arms of the cantilever. 
1 Here we see that the peak temperature at the tip is reduced by $\sim 30{ }^{\circ} \mathrm{C}$ in presence of $2000 \mathrm{ppm}$

2 of isopropanol vapor, while the temperature profile becomes wider. Interestingly, the side

3 arms adjacent to the tip area exhibit an increase in temperature by $\sim 20^{\circ} \mathrm{C}$. It is interesting to

4 note that the current in our TMH sensor increased, i.e. the overall resistance decreased, in

5 presence of isopropanol vapor. Thus, any exothermic reaction between the VOC and air can

6 be ruled out in contrast with hot bead pellistors. ${ }^{[3]}$ In Figure 3d, we show the peak

7 temperature at the tip determined by IR microscopy and also from calibrated Raman

8 spectroscopy, details of which can be found in Section II of Supporting Information. The peak

9 temperature was found to vary from 105 to $330{ }^{\circ} \mathrm{C}$ as the applied voltage bias was increased

10 from 20 to $50 \mathrm{~V}$. The temperature was determined by calibrating the IR camera, and the

11 Raman peak shift using an identical cantilever on a temperature controlled hot plate. The

12 voltage bias was swept from 0 to $50 \mathrm{~V}$ at $5 \mathrm{~V}$ intervals and the steady-state temperature was

13 measured at each bias point, Figure $3 \mathrm{~d}$ shows the $20-50 \mathrm{~V}$ bias range where the sensing

\section{4 experiments are done.}

Considering the temperature profiles shown in Figure 3, we propose a model explaining

17 the response of the TMH sensor in presence of organic vapors. To facilitate our model

18 description, we assume the overall cantilever resistance to consist of several lumped resistors

19 connected in series, each describing the resistance of a specific zone along the arms of the

20 cantilever. These resistances are shown in Figure 3a as $R_{c}$ (contact resistance), $R_{1}$ (side arm

21 resistance), $\mathrm{R}_{2}$ (resistance near the tip) and $\mathrm{R}_{\text {tip }}$ (resistance of the tip region). At lower biases

$22(<10 \mathrm{~V})$, the device exhibits an I-V with almost constant resistance [Figure 1b], indicating

23 the absence of any significant self-heating. At higher biases $(>10 \mathrm{~V}), \mathrm{I}^{2} \mathrm{R}$ loss increases,

24 causing the tip temperature and resistance $R_{\text {tip }}$ to go up. However, the temperatures of $R_{c}$ and

$25 \mathrm{R}_{1}$ do not increase as much as $\mathrm{R}_{\text {tip }}$ with an increase in bias voltage, therefore, these resistances

26 do not change significantly. If a volatile vapor is injected into the test chamber, the vapor 
1 molecules will initially tend to condense in close proximity of the cantilever, but the heated

2 tip region will cause their immediate evaporation. This results in a net loss of thermal energy

3 from this region (defined by resistance $\mathrm{R}_{\text {tip }}$ ) causing its temperature to become lower. The

4 regions of the side arms away from the tip (defined by resistances $R_{2}$ and $R_{1}$ ) do not

5 participate in rapid evaporation of analyte molecules, so the thermal energy loss due to the

6 evaporation of VOCs is not significant there. Since the whole device is under a constant

7 voltage bias, reduction in $R_{\text {tip }}$ will cause an increase in voltage drop across $R_{1}$ and $R_{2}\left(R_{c}\right.$ is

8 likely to be much smaller and the voltage drop across it can be neglected). Since $\mathrm{R}_{2}$ can be

9 expected to be larger than $\mathrm{R}_{1}$ at high temperature, the voltage drop across it is more

10 significant, resulting in a higher temperature rise in that region and consequently larger

11 increase in resistance. Therefore, the total resistance between the arms of the cantilever is

12 affected by the opposite changes in $\mathrm{R}_{\text {tip }}$ and $\mathrm{R}_{2}$, in agreement with the IR image line scan

13 shown in Figure 3c under isopropanol flow. At a moderate bias voltage, i.e. $\sim 10 \mathrm{~V}$, even if a

14 VOC vapor actually causes a reduction in $R_{\text {tip }}$ (by reducing temperature), the increase in $R_{2}$

15 (due to increase in temperature) can compensate for it, since the overall rise in temperature is

16 still low at that bias. Thus, the current can remain unchanged even in presence of a VOC

17 vapor at lower biases. However, as the bias voltage is increased for a given VOC flow,

18 depending on the extent of the temperature drop of $\mathrm{R}_{\text {tip }}$ region (which correlates with the

19 molar latent heat of evaporation, $\Delta \mathrm{H}_{\mathrm{vap}}$ of the VOC), a threshold voltage bias can be reached

20 where the reduction in $\mathrm{R}_{\text {tip }}$ would be more than the increase in $\mathrm{R}_{2}$, thus an increase in current

21 would be observed. Existence of such a threshold voltage, and its dependence on $\Delta \mathrm{H}_{\mathrm{vap}}$, has

22 been experimentally observed, and are shown in Figure 5.

24 To explain the aforementioned physical phenomena, a theoretical model based on heat 25 transfer and Joule heating has been developed. The model is normalized using a number of 26 experimental observations so that the calculated temperature profiles for known conditions 
1 (i.e. bias and analytes) match well with the measured values. The calibrated model can then

2 be used to predict outputs at conditions for which experimental values are difficult to obtain;

3 it can also lead to valuable information on other quantities such as local variation of resistivity

4 (please refer to Section III in supporting information) and so on. The model is based on the

5 following equations for heat transfer (Equation 1 and Equation 3) ${ }^{[27]}$ and Joule heating

6 (Equation 2) which are solved simultaneously and iteratively using finite element method 7 (using COMSOL Multiphysics and MATLAB).

8

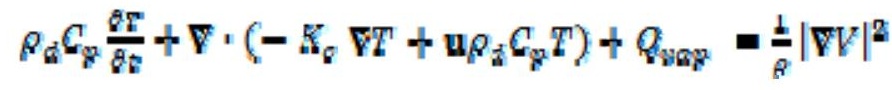

$$
\begin{aligned}
& -\nabla \cdot d\left(\frac{1}{\rho T} \nabla V-i_{r}\right)=0 \\
& \mathbf{n} \cdot\left(K_{Q} F T\right)=q_{0}+h\left(T_{m f}-T\right)+\operatorname{so}\left(T_{m f}^{4}-T^{4}\right)
\end{aligned}
$$

\section{Equation 1 and Equation 2 are solved for different domains of the system, whereas}

Equation 3 is used as a boundary condition at the interface of the solid and surrounding air domains. Here, $\rho_{d}=$ material density, $C_{p}=$ heat capacity, $\mathrm{T}=$ absolute temperature, $\mathrm{K}_{\mathrm{c}}=$ thermal conductivity, $\mathrm{Q}_{\mathrm{vap}}=$ heat loss due to evaporation of analyte molecules (when applicable), $\mathrm{J}_{\mathrm{e}}=$ electrical current density, $\mathrm{V}=$ potential profile, $\rho(\mathrm{T})=$ electrical resistivity as a function of temperature, $\mathbf{n}=$ unit vector normal to the interface, $d=$ thickness of the domain, $\mathrm{q}_{0}=$ heat source inside the domain, $\mathrm{h}=$ temperature dependent coefficient of convection for air, ${ }^{[26]} \mathrm{T}_{\text {inf }}=$ temperature far away from the cantilever, $\varepsilon=$ emissivity of the solid surface, $\sigma=$ Stefan-Boltzmann constant. The temperature dependence of the electrical resistivity is modeled using a least square fit of experimental data (Section III in supporting information), which yields the following empirical relation

$$
P(T)=0.05 T-2,04
$$


1 Since at very low concentration (in parts per million range or below) partial pressure of

2 analyte molecules become very low, they can be considered as ideal gases; and therefore,

3 kinetic theory of gases can be applied to calculate the amount of latent heat taken away from a

4 heated surface by the molecules. For an arbitrary surface area $A_{h}$, amount of heat loss $Q_{\text {vap }}$ per

5 unit time at a given concentration $\mathrm{C}$ and partial pressure $\mathrm{P}_{\mathrm{p}}$ is given by (see Section IV in

6 supporting information for derivation)

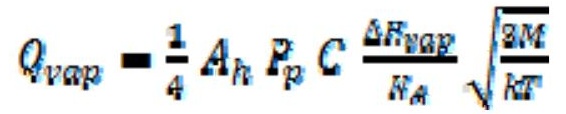

Here, $\Delta \mathrm{H}_{\mathrm{vap}}=$ molar latent heat of evaporation, $\mathrm{N}_{\mathrm{A}}=$ Avogadro's number $\left(6.023 \times 10^{23}\right.$ molecules/mol), $\mathrm{M}=$ molecular mass, $\mathrm{k}=$ Boltzmann's constant $\left(1.38 \times 10^{-23} \mathrm{JK}^{-1}\right)$.

Using the model described above, calculated temperature profiles are obtained and shown in Section III of the Supporting information where it shows a good match to the experimental results shown in Figure 3c. Figure 4a, for $50 \mathrm{~V}$ dc bias, with and without $2000 \mathrm{ppm}$ of isopropanol vapor flow. For simplicity, we assumed that heat was taken away by the molecules from the tip region (see below for definition). The experimentally determined temperature profiles for the similar conditions were presented in Figure 3c, which are reproduced in Figure 4 for comparison. Figure $\mathbf{4 b}$ shows an important phenomenon obtained from the theoretical model that explains a number of subsequent observations. Here, the electrical "path length" of the heated tip region (designated by $\mathrm{R}_{\text {tip }}$ in Figure 3c) is shown for different bias voltages with and without $2000 \mathrm{ppm}$ of isopropanol vapor. In this context, electrical path length refers to the length of the hypothetical resistance $\mathrm{R}_{\text {tip }}$ along the path of the current; which corresponds to the portion of the cantilever that undergoes a negative change in temperature in presence of an analyte vapor. Therefore, in order to calculate the electrical path length of $\mathrm{R}_{\text {tip }}$ (denoted as $\mathrm{L}_{\text {tip }}$ ), temperature profile at each bias point is obtained for no analyte vapor, and for a given analyte vapor at a certain concentration. That is 
1 why, it is important to note that $\mathrm{L}_{\text {tip }}$ strongly depends on the concentration and nature of the

2 analyte. Here in Figure $4 b$, we see that before $14 \mathrm{~V}$ bias, $\mathrm{L}_{\text {tip }}$ is not significant (uniform

3 temperature distribution over most of the cantilever length), indicating negligible effects of

4 analyte vapor on temperature distribution. However, after $14 \mathrm{~V}$ the value of $\mathrm{L}_{\text {tip }}$ starts

5 becoming significant in presence of isopropanol. Although, $\mathrm{L}_{\text {tip }}$ starts to increase after $14 \mathrm{~V}$,

6 the threshold voltage for isopropanol has been experimentally observed to be $\sim 18 \mathrm{~V}$ (please

7 refer to discussions on Figure 5 below), which can be explained based on our earlier

8 discussions, which postulated an increase in $R_{2}$ offsetting any change in $R_{\text {tip }}$ (denoted by $R_{2}$ in

9 Figure 3c) below $18 \mathrm{~V}$. Consequently, the total resistance $\left(\mathrm{R}_{\text {tip }}+\mathrm{R}_{2}\right)$ remains unchanged

10 below $18 \mathrm{~V}$, keeping the tip current unchanged. After $20 \mathrm{~V}, \mathrm{~L}_{\text {tip }}$ increases almost linearly with

11 bias voltage, which indicates the extension of the $\mathrm{R}_{\text {tip }}$ region along the side arms toward the

12 base of the cantilever. Due to the tapered nature of the cantilever, this extension of the $\mathrm{R}_{\text {tip }}$

13 region is expected to saturate as the wider edges of the region would not heat up at the same

14 rate (with change in voltage) as the cantilever tip. This saturation effect of $\mathrm{L}_{\text {tip }}$ can be clearly

15 seen in Figure $4 \mathrm{~b}$ above $40 \mathrm{~V}$. This behavior has important consequences on device sensing

16 performance at higher bias voltages which will be discussed below in relation to Figure 5 and

17 Figure 6.

Figure 5a shows the percentage change in current caused by dilute vapor (2000 ppm molar concentration) of four different VOCs: isopropanol, methanol, hexane and acetone, as 21 the applied voltage bias was varied from 15 to $50 \mathrm{~V}$. Seven readings were averaged at each 22 bias point and a 5-points moving average filtering was performed to ensure better consistency 23 and noise reduction. From the measured data points, the approximate uncertainty in the 24 threshold voltage was estimated to be $\pm 0.1 \mathrm{~V}$. As can be seen from Figure 5a, each analyte 25 corresponds to a distinct threshold voltage $\left(\mathrm{V}_{\text {th }}\right)$ below which it does not cause any noticeable 26 change in current. Although the current nearly saturates after $\sim 20 \mathrm{~V}$, tip temperature still 
1 keeps on increasing with an increased bias due to the higher power dissipation (i.e self-

2 heating), which is why we still need to increase the voltage bias to observe the sensor

3 response despite having a fairly constant current through the device. In the present work, $\mathrm{V}_{\text {th }}$

4 is arbitrarily defined using a current magnitude change of $0.1 \%$ or higher (considering our

5 noise level of $0.04 \%$ and a signal to noise ratio of $>2.5$ ) in presence of an analyte; however,

6 for a low noise environment, it can be defined at a lower value as well. To determine the $\mathrm{V}_{\text {th }}$

7 from our measurements we use the following methodology; if within a voltage range of $0.2 \mathrm{~V}$

8 the change in current is at least $0.1 \%$ (in presence of an analyte vapor), then the mid-value of

9 that voltage range is defined as $\mathrm{V}_{\text {th }}$. The threshold voltage for each analyte was observed to be

10 very consistent over multiple sets of experiments performed in a period of more than six

11 month utilizing several identical devices. To verify if a generic correlation indeed exists

12 between $\Delta \mathrm{H}_{\text {vap }}$ and $\mathrm{V}_{\text {th }}$, as predicted by our model, threshold voltages of seven different

13 analytes with latent heat varying over a wide range were determined. The plot of $\mathrm{V}_{\text {th }}$ versus

$14 \Delta \mathrm{H}_{\text {vap }}$ is shown in Figure 5b, where an excellent linear correlation is observed. This is in

15 agreement with our proposed model (please refer to the explanation of Figure 3, where a

16 correlation between $\mathrm{V}_{\text {th }}$ and $\Delta \mathrm{H}_{\text {vap }}$ was predicted), and clearly indicates that such a well-

17 defined correlation can be utilized to perform selective detection of VOC vapors from their

18 unique threshold voltages. It should be noted here that the temperature of the tip (measured

19 using the IR camera) at the threshold voltage was always lower than the auto-ignition

20 temperature for all the seven VOCs studied, ruling out any combustion related effects. Also,

21 as mentioned above, no effect of water vapor of similar molar concentration (2000 ppm) was

22 observed (see Figure 2) even up to a bias of $50 \mathrm{~V}$, which is much higher than the expected $\mathrm{V}_{\text {th }}$

23 of $23 \mathrm{~V}$ based on its $\Delta \mathrm{H}_{\mathrm{vap}}$ of $40 \mathrm{~kJ} / \mathrm{mol}$. This, therefore, rules out any interference from water

24 vapor in realistic sensing environments. To investigate any dependence of the $\mathrm{V}_{\text {th }}$ on analyte

25 concentration, detection was performed with much diluted (down to $\sim 50 \mathrm{ppm}$ concentration)

26 vapor of isopropanol, ethanol and toluene. The $\mathrm{V}_{\text {th }}$ values were found to increase only by 
$1 \sim 0.75 \mathrm{~V}$ as the vapor concentrations were reduced 40 fold from 2000 to $50 \mathrm{ppm}$ (discussed in

2 detail in Figure 6). This indicates that $\mathrm{V}_{\text {th }}$ remains effectively constant, and thus can be used

3 to reliably identify VOCs over their significant detection range, especially for indoor

4 environments. ${ }^{[28]}$

Since III-Nitrides have strong polarization properties, it is interesting to explore possible 7 correlations between the magnitude of the molecular dipole moment of the analyte VOC and 8 the magnitude of current change caused at a fixed applied bias. Figure $\mathbf{5} \mathrm{c}$ shows the 9 percentage change in current for seven VOCs (measured at a constant bias of $50 \mathrm{~V}$ ) plotted 10 against their dipole moment. We find that as the dipole moment of the analyte VOC increases, 11 the response magnitude also increases, which is expected since the molecules with higher 12 dipole moment are expected to have stronger interaction with the highly polar AlGaN 13 surface. ${ }^{[29]}$ This would cause a larger change in tip temperature and hence in the overall 14 magnitude of the current change. Although the physical mechanism of molecular interaction 15 is unclear to us at present, it is possible that the polar AlGaN surface is responsible for 16 attracting the polar VOC molecules toward it, (which is dependent on the dipole moment) and 17 allowing them to condense to a certain extent. When the condensed molecules evaporate, the 18 latent heat taken away (and hence the response magnitude) is therefore also proportional to 19 the molecular dipole moment. For example, acetone, in spite of having a rather low $\Delta \mathrm{H}_{\mathrm{vap}}$, 20 still caused the largest change in current at $50 \mathrm{~V}$, since its dipole moment is the highest among 21 all the analytes studied. If multiple analytes have very similar dipole moments, the change in 22 current depends on their latent heat of evaporation, which is clearly evident from Figure 5b 23 with respect to isopropanol, ethanol and methanol. Here all three have similar dipole moments 24 (in the range $1.6-1.7 \mathrm{D}$ ), so the one with highest latent heat of evaporation (isopropanol) 25 caused the largest change in current. We would like to point out here that the effect of 26 polarization is clearly observed only at high bias voltages. At low bias voltages, closer to the 
$1 \mathrm{~V}_{\text {th }}$ for a particular analyte, the response is primarily controlled by the temperature

2 distribution of the tip (please refer to earlier discussions). Although polarization effect is also

3 present for this bias range, it is insignificant compared to the other effects. However, at high

4 bias voltages (i.e. over $45 \mathrm{~V}$, which is much higher than the $\mathrm{V}_{\text {th }}$ of analytes considered here),

5 effective surface area of the hot zone near the tip tends to saturate (temperature still keeps

6 increasing though), allowing the surface polarization to play a dominant role in controlling the

7 heat transfer to the analyte molecules, and consequently the device response. The power

8 dissipation and the tip temperature keep on increasing as the bias voltage goes up; however

9 the device response has been found to be stable at $50 \mathrm{~V}$ bias in spite of significant self-heating.

$10 \underline{\text { In regular ambient environment, the devices have been tested for up to } 90 \mathrm{~V} \text { bias repeatedly }}$

11 for several days and it has been concluded that a $50 \mathrm{~V}$ bias could certainly be used for reliable

12 and repeatable results. While a smaller bias $(18-32 \mathrm{~V})$ is sufficient for detecting $\mathrm{V}_{\text {th }}$, a larger

13 voltage bias of $50 \mathrm{~V}$ is required to observe the other modality of the sensor that is related to

14 the dipole moment of the analytes.

15

In order to establish the utility of $\mathrm{V}_{\text {th }}$ for uniquely identifying an analyte, we performed systematic measurements of device characteristics as a function of analyte concentration. The results are shown in Figure 6 for three different analytes, isopropanol, ethanol and toluene, which were chosen to cover a wide range of $\Delta \mathrm{H}_{\text {vap. }}$. As observed from Figure 6a, the $\mathrm{V}_{\text {th }}$ values corresponding to different analytes changed only slightly $(0.6-0.75 \mathrm{~V})$ when their concentration was reduced from $2000 \mathrm{ppm}$ to $50 \mathrm{ppm}$. As the analyte concentration is increased, more molecules are able to interact with the cantilever, thus causing the $V_{\text {th }}$ to reduce. Although, the relatively small magnitude of change in $\mathrm{V}_{\text {th }}$ is unclear to us at this point, nonetheless, $\mathrm{V}_{\text {th }}$ is obviously an important parameter that can be utilized in a practical sensor to uniquely identify different VOCs over a specified range of concentration. 
1 In order to identify the threshold voltage, it is also important to observe the dependence

2 of current magnitude change at the threshold voltage. In the post-threshold biasing region,

3 more and more analyte molecules can interact with the heated cantilever tip as the effective

4 area of the hot zone (corresponding to $\mathrm{R}_{\text {tip }}$ ) increases with bias voltage. Increased temperature

5 also increases the convection flow, creating a low pressure region in the vicinity of the heated

6 tip of the cantilever. This causes faster circulation of analyte vapor around the cantilever tip

7 resulting in more molecules to interact with the tip per unit time. Therefore, just above the

8 threshold voltage, for the same analyte concentration, current is mostly governed by the

9 effective area of the heated tip region as shown in Figure 6b, where the analyte with the

10 lowest threshold voltage has the lowest threshold current (see definition below) because of the

11 smaller effective area of hot zone at that voltage. Extrapolating all the curves towards low

12 concentration gives a noise limited resolution of $\sim 5 \mathrm{ppm}$. The threshold current is calculated

13 by first taking a voltage bias which is $0.1 \mathrm{~V}$ above the threshold voltage. Then the change in

14 current is measured in presence of an analyte vapor of a certain concentration. The threshold

15 current response (i.e. normalized change in current) is then defined by the following relation

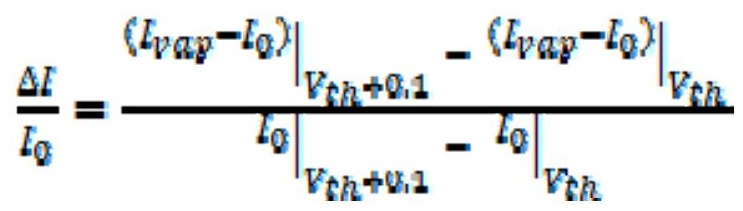

Here, $I_{v a p}$ and $I_{0}$ are the currents measured with and without analyte vapor, respectively. The same is also done for dry UHP $\mathrm{N}_{2}$ instead of analyte to obtain the background response for the carrier gas (dry UHP $\mathrm{N}_{2}$ ) and the noise signal, which was found to be within $0.04 \%$ over the entire bias range (15 to $35 \mathrm{~V})$. Figure $6 \mathrm{~b}$ also shows a linear relation between threshold current response with logarithm of concentration, which is common for many sensors.

Above the threshold voltage, percentage change of current in presence of a particular VOC depends on the concentration of analyte vapor. We define sensitivity of the sensor as the percent change in current for one decade change in analyte concentration; change in current is 
1 calculated at threshold voltage using Equation 6. We denote this sensitivity by S, which is

2 calculated using the following expression,

3

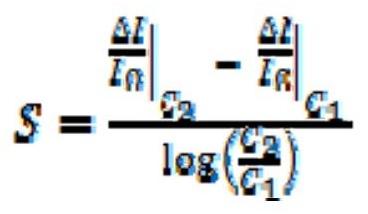

4 where $\mathrm{C}_{2}$ and $\mathrm{C}_{1}$ are the vapor concentrations $\left(\mathrm{C}_{2}>\mathrm{C}_{1}\right)$. Table 1 shows the sensitivity of the

$5 \mathrm{TMH}$ sensor for 50 and $2000 \mathrm{ppm}$ concentration of isopropanol, ethanol, toluene and acetone

6 vapors. We find that an analyte with a higher threshold voltage leads to higher detection

7 sensitivity, which is also evident in Figure 6b, where the slope of the fitted line is higher for

8 such an analyte. Table 1 also lists the rise and fall times of the sensor for different analytes. It

9 is observed that the rise/fall times are similar for all four analytes at high concentration (2000

$10 \mathrm{ppm})$, however at low concentration $(50 \mathrm{ppm})$ they start to deviate quite significantly (by up

11 to $10 \mathrm{~s}$ ). The rise/fall time decreases by several seconds as the voltage bias (i.e. cantilever

12 temperature) goes up.

14 We also estimated the limit of detection (LOD, in terms of vapor concentration), from our

15 simulation results, corresponding to the threshold current response assuming it to vary over

16 the range of $0.001 \%$ and $0.1 \%$. The results are presented in Figure 7 a for 3 different analytes.

17 Interestingly, all the responses can be seen to merge asymptotically at low concentration,

18 which is supported by our experimental results, as shown in Figure 6b, where the

19 extrapolated curves also intersect at low concentration. Such an intersection of extrapolated

20 responses for various analytes in however expected, since at very low concentration (i.e.

21 below detection level), the various analyte vapors would produce very similar response to the

22 carrier gas alone (i.e. $\mathrm{N}_{2}$ ), which is basically defined by the system noise. The LOD for

23 different analytes at $0.001 \%$ threshold current response are tabulated in Figure $7 \mathrm{~b}$. 
The TMH sensors used in this work were studied over a period of at least a year, both in

2 open air and in a closed chamber; and were subjected to much higher applied biases (up to

$3 \sim 90 \mathrm{~V}$ ) than those reported here. However, no noticeable change in device characteristics was

4 observed in this time frame, which underscores high measurement reliability as well as

5 thermal and chemical stability of these sensors.

\section{4. Conclusions}

8 In conclusion, we have demonstrated novel $\mathrm{AlGaN} / \mathrm{GaN}$ heterostructure based triangular

9 microcantilever heaters, and utilized them to perform unique detection of VOCs down to the

10 low ppm (down to 5 pm limited by noise) range with high specificity, while operating below

11 their auto-ignition temperatures. It has been observed that for a particular VOC, over a certain

12 concentration range, a well-defined threshold voltage exists, which varies linearly with the

13 molar latent heat of evaporation. The response magnitude for a VOC at a fixed voltage bias

14 was found to be related to the molecular dipole moment. A simple model has been proposed

15 to explain the response of the sensor to different VOCs.

16

17

18

19

20

21

22

23

24

25

26

\section{Acknowledgements}

Financial support for this work from National Science Foundation (Grants\# ECCS-0846898, ECCS-1348166 and IIP-1343437), are thankfully acknowledged. 


\section{References:}

2 [1] H. Guo, S. C. Lee, L. Y. Chan, and W. M. Li, Risk assessment of exposure to volatile organic compounds in different indoor environments, Environ. Res. 94 (1), 57-66 (2004)

[2] H. Singh, Y. Chen, A. Staudt, D. Jacob, D. Blake, B. Heikes, and J. Snow, Evidence from the Pacific troposphere for large global sources of oxygenated organic compounds, Nature 410 (6832), 1078-1081 (2001)

[3] M. Gall, The Si-planar-pellistor array, a detection unit for combustible gases, Sens. Actuators B 16 (1), 260-264 (1993)

[4] M.-T. Ke, M.-T. Lee, C.-Y. Lee, and L.-M. Fu, A MEMS-based benzene gas sensor with a self-heating $\mathrm{WO}_{3}$ sensing layer, Sensors 9, 2895-2906 (2009)

[5] C. J. Martinez, B. Hockey, C. B. Montgomery, and S. Semancik, Porous tin oxide nanostructured microspheres for sensor applications, Langmuir, 21 (17), 7937-7944 (2005)

[6] K. D. Benkstein, and S. Semancik, Mesoporous nanoparticle $\mathrm{TiO}_{2}$ thin films for conductometric gas sensing on microhotplate platforms, Sens. Actuators B 113(1), 445-453 (2006)

[7] R. Artzi-Gerlitz, K. D. Benkstein, D. L. Lahr, J. L. Hertz, C. B. Montgomery, J. E. Bonevich, S. Semancik, and M. J. Tarlov, Fabrication and gas sensing performance of parallel assemblies of metal oxide nanotubes supported by porous aluminum oxide membranes, Sens. Actuators B 136 (1), 257-264 (2009).

[8] M. Qazi, G. Koley, S. Park, T. Vogt, $\mathrm{NO}_{2}$ detection by adsorption induced work function changes in $\operatorname{In}_{2} \mathrm{O}_{3}$ thin films, Appl. Phys. Lett. 91 (4), 043113 (2007)

[9] M. Qazi and G. Koley, $\mathrm{NO}_{2}$ detection using microcantilever based potentiometry, Sensors 8, 7144 (2008)

[10] G. Koley, M. G. Spencer, H. R. Bhangale, Cantilever effects on the measurement of electrostatic potentials by scanning Kelvin probe microscopy, Appl. Phys. Lett. 79 (4), 545-547 (2001)

[11] M. Qazi, N. DeRoller, A. Talukdar, G. Koley, III-V Nitride based piezoresistive microcantilever for sensing applications, Appl. Phys. Lett. 99 (19), 193508 (2011) 
1 [12] Y. J. Tang, J. Fang, X. H. Xu, H. F. Ji, G. M. Brown, and T. Thundat, Detection of femtomolar concentrations of $\mathrm{HF}$ using an $\mathrm{SiO}_{2}$ microcantilever, Anal. Chem. 76, $2478(2004)$

[13] G. Koley, M. Qazi , L. Lakshmanan, and T. G. Thundat, Gas sensing using electrostatic force potentiometry, Appl. Phys. Lett. 90 (17), 173105 (2007)

[14] L.A Pinnaduwage, T Thundat, J.E Hawk, D.L Hedden, P.F Britt, E.J Houser, S Stepnowski, R.A McGill, D Bubb, Detection of 2,4-dinitrotoluene using microcantilever sensors, Sens. Actuators B 99 (2-3), 223-229 (2004)

[15] Z. Hu, T. Thundat, and R. J. Warmack, Investigation of adsorption and absorptioninduced stresses using microcantilever sensors, J. Appl. Phys. 90 (1), 427-431 (2001)

[16] C. Canetta and A. Narayanaswamy, Sub-picowatt resolution calorimetry with a bimaterial microcantilever sensor, Appl. Phys. Lett. 102 (10), 103112 (2013)

[17] J. O. Liu, S. Somnath, and W. P. King, Heated atomic force microscope cantilever with high resistivity for improved temperature sensitivity, Sens. Actuators A 201, 141$147(2013)$

[18] J. Lee, T. L. Wright, M. R. Abel, E. O. Sunden, A. Marchenkov, S. Graham, and W. P. King, Thermal conduction from microcantilever heaters in partial vacuum, J. Appl. Phys. 101(1), 014906 (2007)

[19] J. Lee and W. P. King, Microcantilever hotplates: Design, fabrication, and characterization, Sens. Actuators A 136, 291-298 (2007)

[20] J. Lee, T. Beechem, T. L. Wright, B. A. Nelson, S. Graham, and W. P. King, Electrical, thermal, and mechanical characterization of silicon microcantilever heaters, J. Microelectromech. Syst. 15(6), (2006)

[21] L. A. Pinnaduwage, V. Boiadjiev, J. E. Hawk, and T. Thundat, Sensitive detection of plastic explosives with self-assembled monolayer-coated microcantilevers, Appl. Phys. Lett. 83 (7), 1471-1473 (2003)

[22] A. Loui, D. J. Sirbuly, S. Elhadj, S. K. McCall, B. R. Hart, and T. V. Ratto, Detection and discrimination of pure gases and binary mixtures using a dual-modality microcantilever sensor, Sens. Actuators A 159(1), 58-63 (2010) 
1 [23] S. Tétin, B. Caillard, F. Ménil, H. Debéda, C. Lucat, C. Pellet, and I. Dufour,

\section{8} 20 21 Modeling and performance of uncoated microcantilever-based chemical sensors, Sens. Actuators B 143(2), 555-560 (2010)

[24] G. Koley, M. G. Spencer, Surface potential measurements on GaN and AlGaN/GaN heterostructures by scanning Kelvin probe microscopy, J. Appl. Phys. 90 (1), 337-344 (2001)

[25] A. Talukdar, M. Qazi, and G. Koley, High frequency dynamic bending response of piezoresistive GaN microcantilevers, Appl. Phys. Lett. 101 (25), 252102 (2012)

[26] A. Talukdar, and G. Koley, Impact of biasing conditions on displacement transduction by III-Nitride microcantilevers, IEEE Elec. Dev. Lett. 35 (12), 1299-1301 (2014)

[27] F. P. Incropera and D. P. DeWitt, Fundamentals of Heat and Mass Transfer, 4th ed, John Wiley \& Sons, New York, 1996.

[28] S. K. Brown, M. R. Sim, M. J. Abramson, and C. N. Gray, Concentrations of volatile organic compounds in indoor air-a review, Indoor Air 4(2), 123-134 (1994)

[29] R. Neuberger, G. Müller, O. Ambacher, and M. Stutzmann, High-electron-mobility AlGaN/GaN transistors (HEMTs) for fluid monitoring applications, Phys. Status Solidi A 185(1), 85-89 (2001)

9 
2 Table Caption

3 Table 1. Sensitivity, rise time and fall time for different analytes at threshold voltage.

4

5

6 Figure Caption

8 Figure 1. (a) I-V characteristics of V-shaped micro cantilever heater sensor. Inset shows the 9 SEM image of the device. (b) Change of power and resistance with bias voltage. Photograph of a wire-bonded device mounted on a dual inline package (DIP) is shown in the inset.

Figure 2. (a) Response of the device to $2000 \mathrm{ppm}$ of hexane and water vapor at $50 \mathrm{~V}$ dc bias. In presence of hexane vapor flow, the current increased by about $1.84 \%$; whereas water vapor

14 did not cause any change in current. (b) Response of the device to $400 \mathrm{ppm}$ of hexane in two 15 consecutive cycles to demonstrate the repeatability of the sensor behavior. Each time, there was a change of about $1.1 \%$ in current; rise time and fall time are, however, longer than that 17 in (a) as concentration is five times lower.

Figure 3. (a) False color infrared image of the cantilever shown in Figure 1a under $50 \mathrm{~V}$ bias.

20 The outline of the cantilever is super-imposed on the thermal image. Lumped resistors $R_{c}, R_{1}$, $21 \mathrm{R}_{2}$ and $\mathrm{R}_{\text {tip }}$ represent different regions of the device and are used to model it. (b) The 22 cantilever under same bias, but in presence of isopropanol vapor with a molar concentration 23 of 2000 ppm. (c) Line scan of temperature along the cantilever as shown in (a) and (b). Inset 24 shows the equivalent circuit based on the model resistors shown in (a). (d) Temperature 25 calibration of the heated cantilever with different bias using calibrated IR microscopy and 26 Raman spectroscopy with identical linear trend. 
2 Figure 4. (a) Simulated temperature profiles for the heated cantilever in intrinsic condition

3 and in presence of 2000 ppm of isopropanol vapor at $50 \mathrm{~V}$ bias. (b) Electrical path length of

4 the heated tip resistance (denoted by $\mathrm{R}_{\text {tip }}$ ), $\mathrm{L}_{\text {tip }}$ as a function of bias voltage for $2000 \mathrm{ppm}$ of 5 isopropanol vapor.

7 Figure 5. (a) Composite plots of normalized change in current (\%) with change in voltage for 8 isopropanol, methanol, acetone and hexane at $2000 \mathrm{ppm}$ molar concentration; showing a 9 distinct voltage for each analyte below which there is no change in current. (b) Dipole 10 Moment $(\mu)$ and latent heat of evaporation $\left(\Delta \mathrm{H}_{\mathrm{vap}}\right)$ for different volatile organic compounds 11 (VOCs). (c) Linear relation between threshold voltage of sensing $\left(\mathrm{V}_{\text {th }}\right)$ and latent heat of 12 evaporation $\left(\Delta \mathrm{H}_{\mathrm{vap}}\right)$ for different VOCs at molar concentrations of 100, 400 and $2000 \mathrm{ppm}$. $13 \mathrm{~V}_{\text {th }}$ is observed to vary slightly with the change of concentration. (d) Correlation between 14 normalized change in current (\%) at $50 \mathrm{~V}$ and dipole moment $(\mu)$ for VOCs at 100,400 and $152000 \mathrm{ppm}$ molar concentration. Linear relation for each analyte concentration has been observed; least square fit parameters for the fitted straight lines are given in the table in the 17 inset.

Figure 6. (a) Dependence of $\mathrm{V}_{\text {th }}$ on molar concentration of isopropanol, ethanol and toluene. 20 As it is found out, change in threshold voltage is no more than $0.75 \mathrm{~V}$ for all three of these 21 analytes. (b) Change in current at threshold voltage for the same analytes. This limits the 22 detectability of the threshold voltage as concentration goes down. Extrapolated curves 23 indicate a noise limited resolution of $5 \mathrm{ppm}$ with a sensor noise level of $0.04 \%$ change in 24 output current. 
1 Figure 7. (a) Theoretical lowest concentration of isopropanol, ethanol and acetone that can be 2 detected for a given change in current at threshold voltage. System noise level of $0.04 \%$ has 3 been shown here, indicating a threoretical noise limited resolution below 2 ppm. (b) A 4 compilation of theoretical lower limits for various analytes at $0.001 \%$ change in current at 5 threshold voltage.

6

7

8

9

10 
Ifat Jahangir received the B.Sc. degree in Electrical and Electronic Engineering from Bangladesh University of Engineering and Technology (BUET) in 2011. He has been pursuing his Ph.D. degree in Electrical Engineering at the University of South Carolina, since 2012. His research interests include development and characterization of III-Nitride and 2D materials based devices for electronic and sensing applications. He is a graduate student member of IEEE, IEEE Electron Devices society and Optical Society of America (OSA).

Dr. Ehtesham Bin Quddus was born in Dhaka, Bangladesh in 1983. He received his B.Sc. degree in Electrical and Electronic Eng. from Bangladesh University of Engineering and Technology (BUET) in 2006 and Ph.D. degree in Electrical Engineering from University of South Carolina in 2013. Since then, he has been with Intel Corporation, Hillsboro, Oregon, where he is currently working as a Process Engineer in Intel's $14 \mathrm{~nm}$ process node.

Dr.GoutamKoley received his B. Tech. degree from the Indian Institute of Technology, Kharagpur, in 1998, M.S. degree from the University of Massachusetts Lowell, in 1999, and Ph.D. degree from Cornell University in 2003. Immediately after his graduation, he joined the department of Electrical Engineering at the University of South Carolina as an Assistant Professor, and was promoted to the rank of Associate Professor with tenure in 2009. He joined Clemson University in 2014 as a Professor in the Department of Electrical and Computer Engineering. His current research interests include III-Nitride MEMS based chemical, physical and biological sensors and two-dimensional materials based nanoscale sensors and electronic devices. Dr. Koley has published 56 journal articles, coauthored 2 book chapters and presented more than 125 conference talks and seminars. He also has 3 issued patents with several others pending. He is a co-founder of three start-up companies, a senior member of the IEEE and a member of the APS. 
Table 1.Sensitivity, rise time and fall time for different analytes at threshold voltage.

\begin{tabular}{|c|c|c|c|c|c|c|c|c|c|c|}
\hline \multirow[b]{2}{*}{ Analyte } & \multicolumn{3}{|c|}{$\mathrm{V}_{\text {th }}(\mathrm{V})$} & \multirow[b]{2}{*}{ Sensitivity (\%) } & \multicolumn{3}{|c|}{ Rise Time (s) } & \multicolumn{3}{|c|}{ Fall Time (s) } \\
\hline & $\begin{array}{l}2000 \\
\text { ppm }\end{array}$ & $\begin{array}{l}1000 \\
\text { ppm }\end{array}$ & $\begin{array}{c}50 \\
\text { ppm }\end{array}$ & & $\begin{array}{c}2000 \\
\mathrm{ppm}\end{array}$ & $\begin{array}{l}1000 \\
\mathrm{ppm}\end{array}$ & $\begin{array}{c}50 \\
\mathrm{ppm}\end{array}$ & $\begin{array}{l}2000 \\
\mathrm{ppm}\end{array}$ & $\begin{array}{l}1000 \\
\text { ppm }\end{array}$ & $\begin{array}{c}50 \\
\text { ppm }\end{array}$ \\
\hline Isopropanol & 18.0 & 18.2 & 18.7 & 0.172 & 15 & 21 & 54 & 22 & 30 & 78 \\
\hline Ethanol & 23.0 & 23.2 & 23.75 & 0.224 & 14 & 21 & 51 & 19 & 27 & 72 \\
\hline Toluene & 27.0 & 27.1 & 27.5 & 0.251 & 17 & 24 & 58 & 23 & 32 & 83 \\
\hline Acetone & 29.0 & 29.1 & 29.6 & 0.256 & 14 & 22 & 53 & 20 & 27 & 75 \\
\hline
\end{tabular}



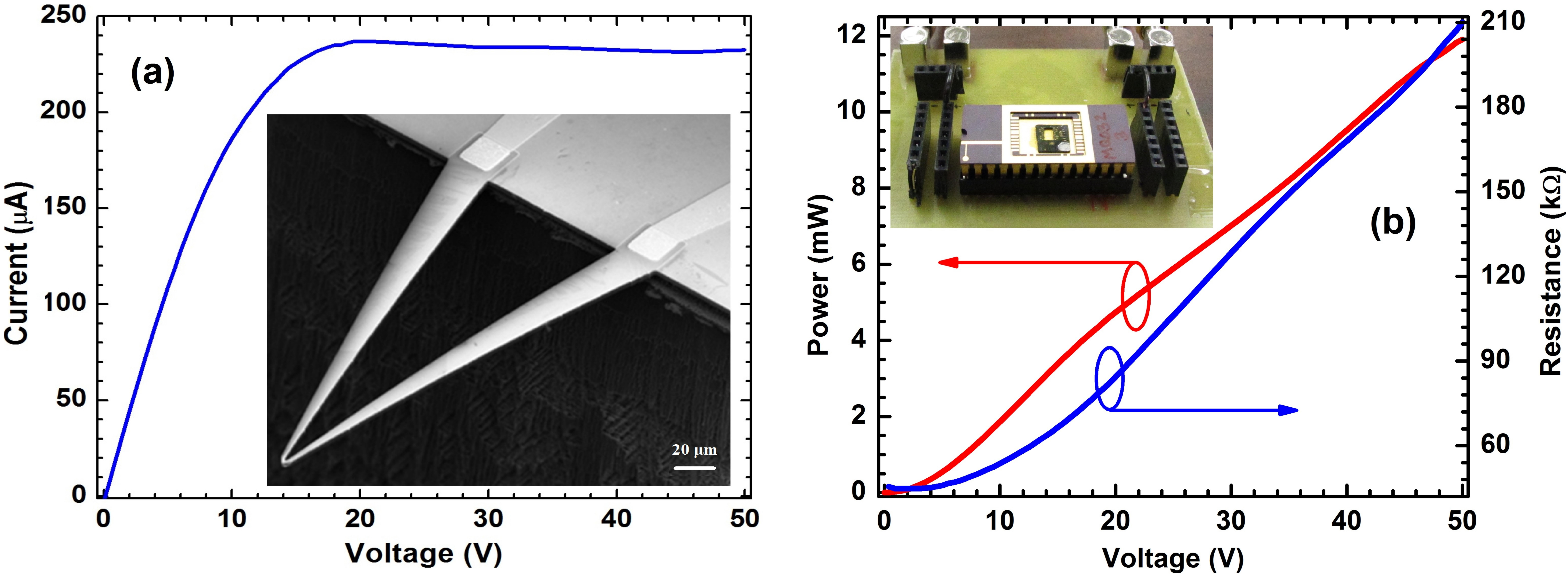

Figure 1 

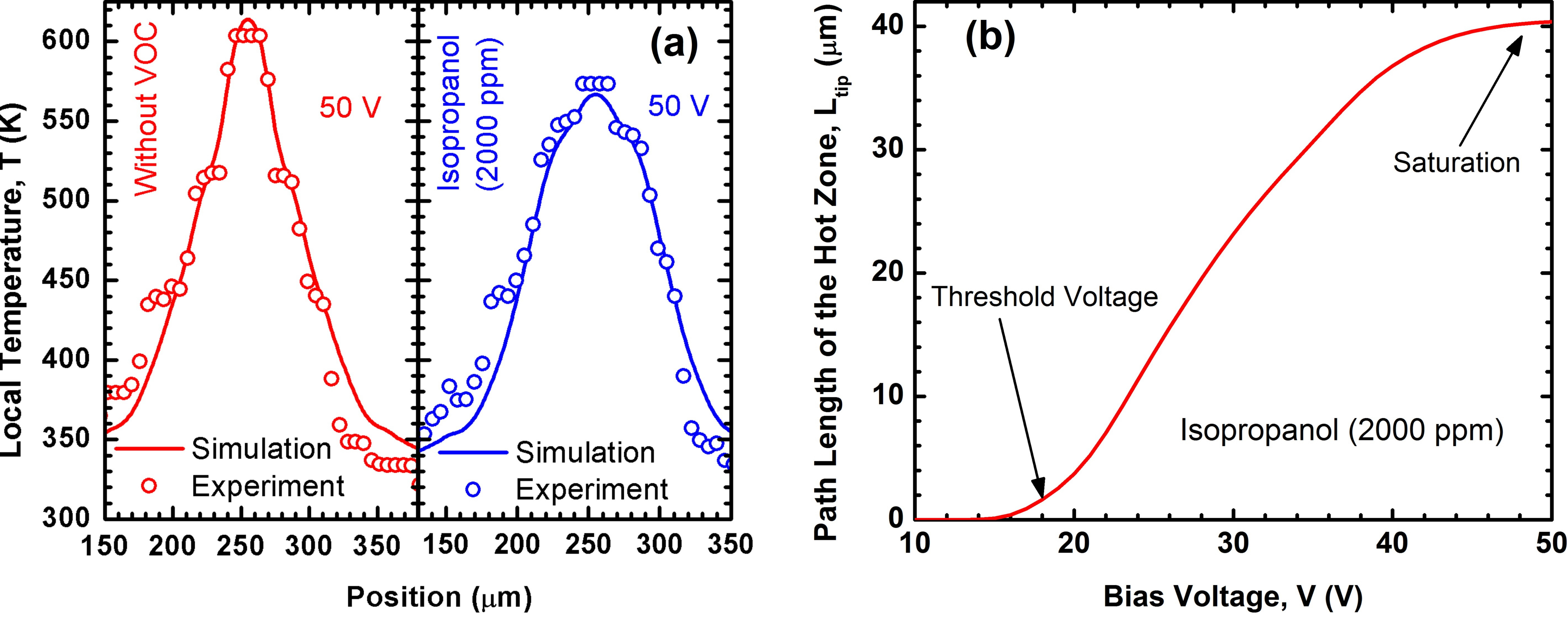

\section{Figure 4}




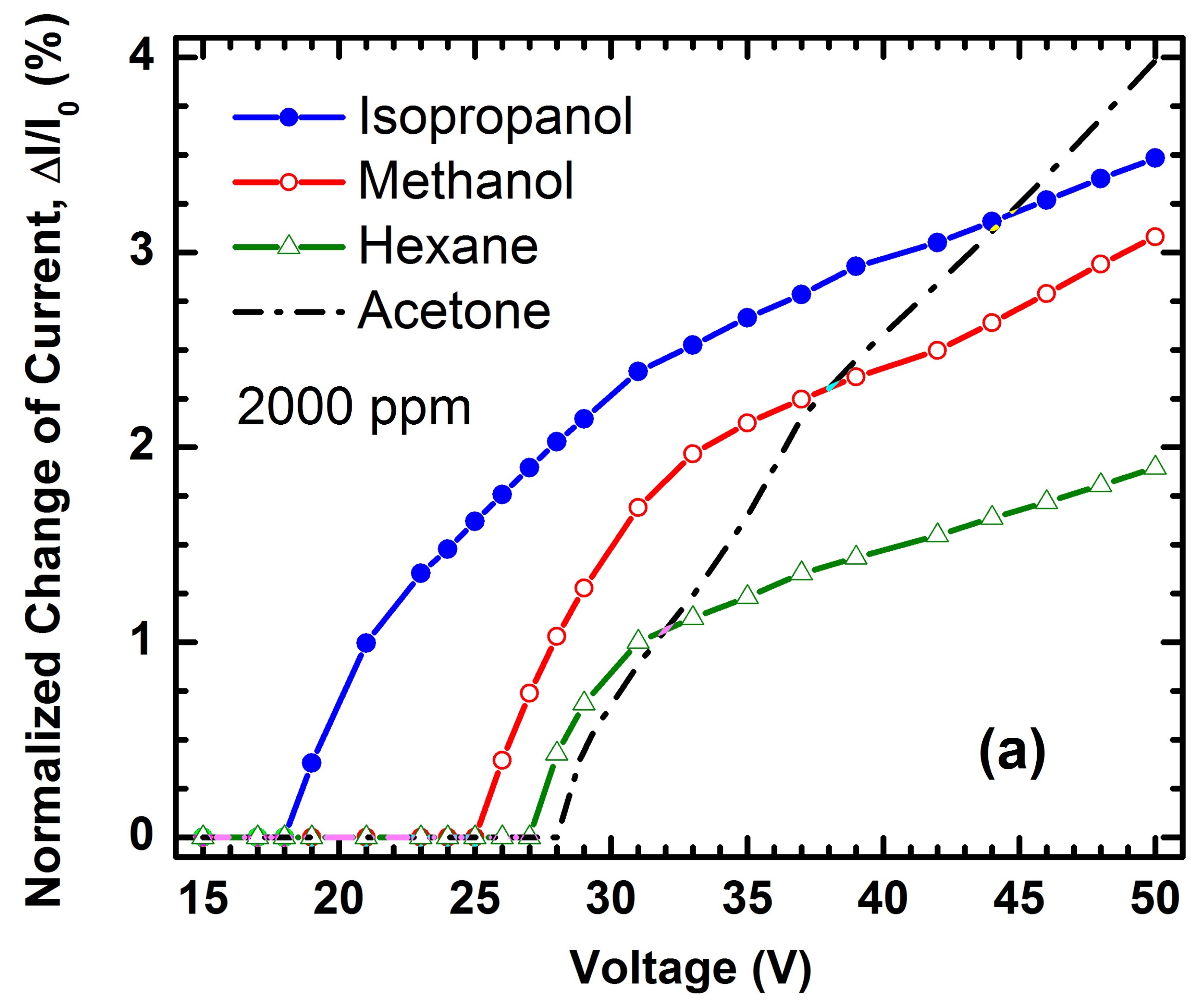

(b) Dipole Moment $(\mu)$ and Latent Heat of Evaporation $\left(\Delta \mathrm{H}_{\mathrm{vap}}\right)$ of VOCs

\begin{tabular}{|c||c||c|}
\hline Analyte & $\boldsymbol{\mu}(\mathrm{D})$ & $\Delta \mathbf{H}_{\text {vap }}(\mathrm{kJ} / \mathrm{mol})$ \\
\hline Hexane & 0.08 & 31.5 \\
\hline Toluene & 0.36 & 32.3 \\
\hline Isopropanol & 1.66 & 45.4 \\
\hline Ethanol & 1.69 & 38.6 \\
\hline Methanol & 1.7 & 35.3 \\
\hline Acetone & 2.69 & 30.5 \\
\hline Diethyl Ether & 1.15 & 27.3 \\
\hline
\end{tabular}

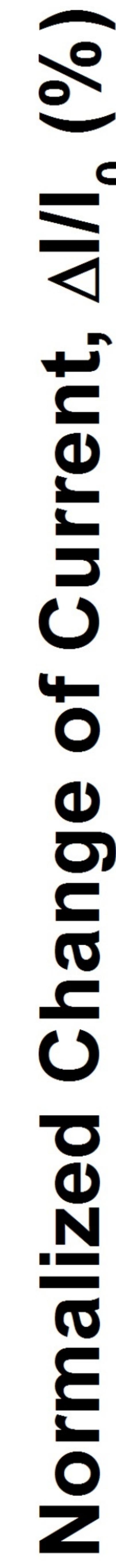
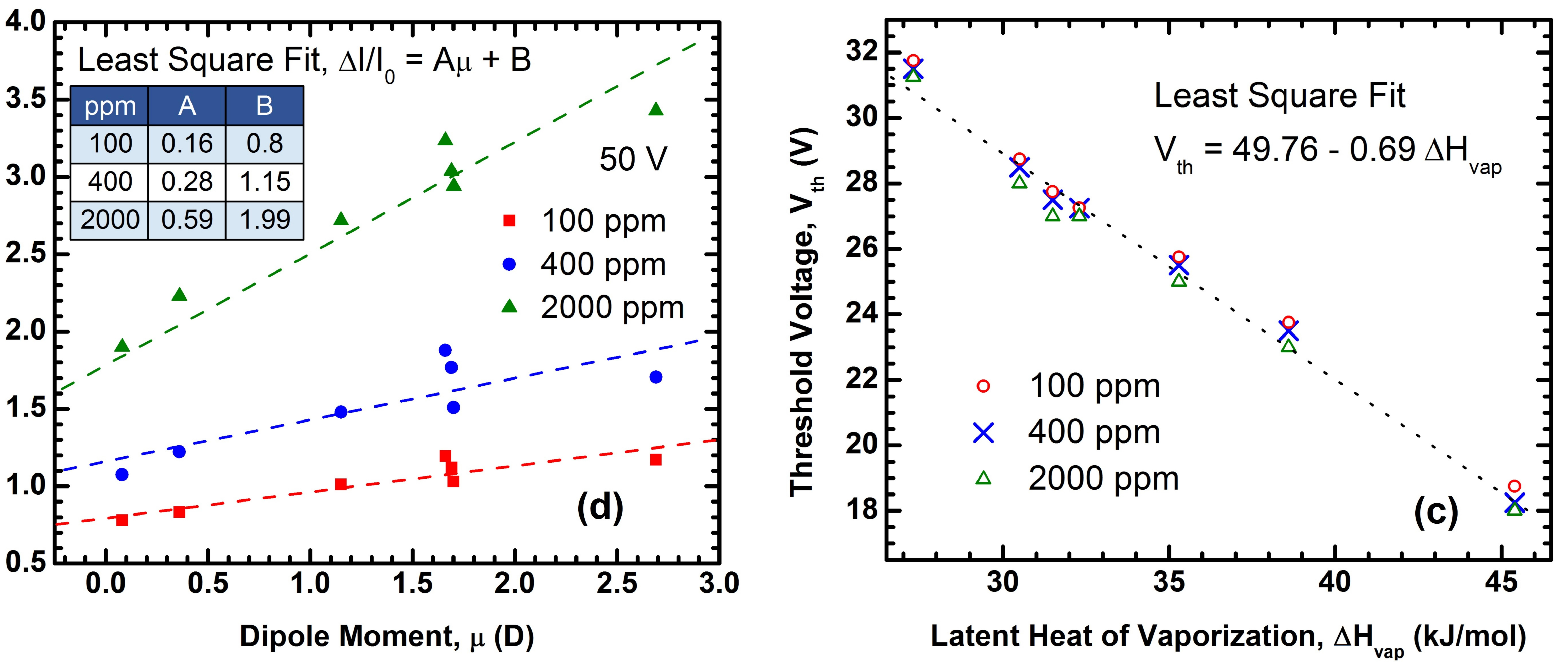

Figure 5 

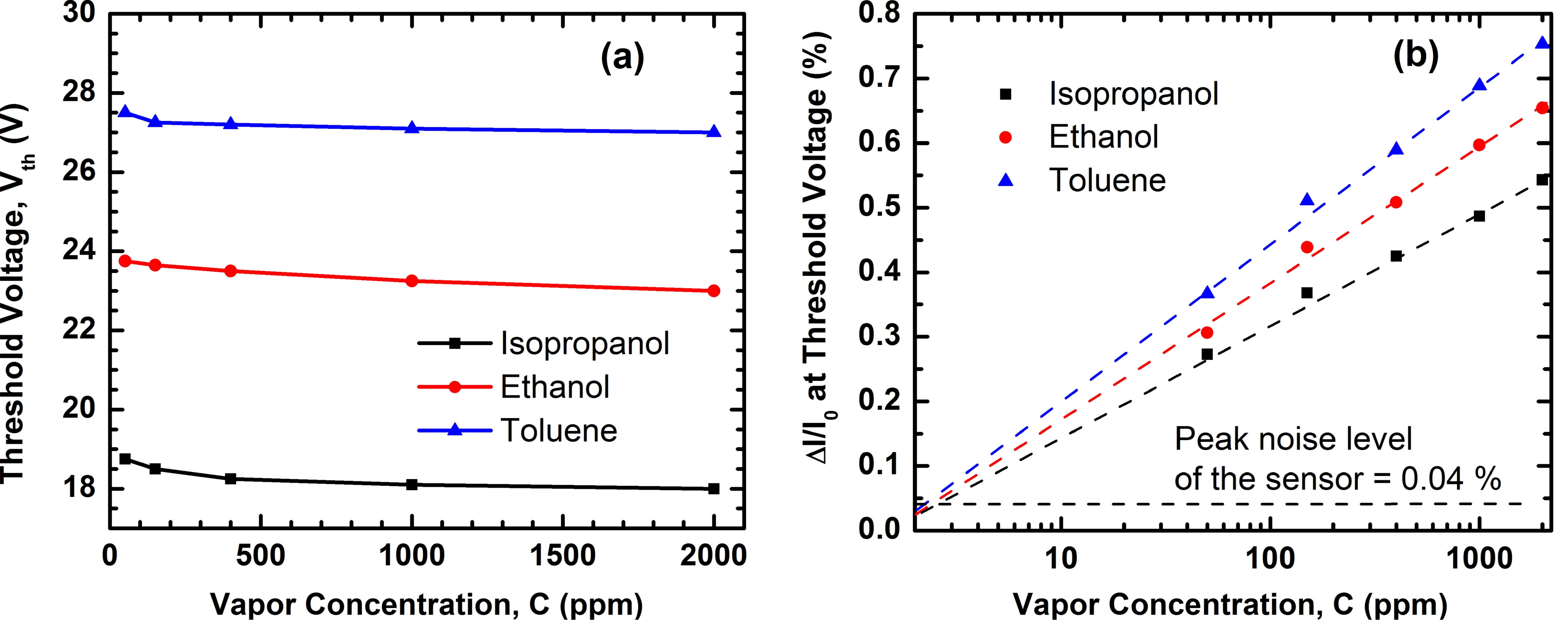

Figure 6 


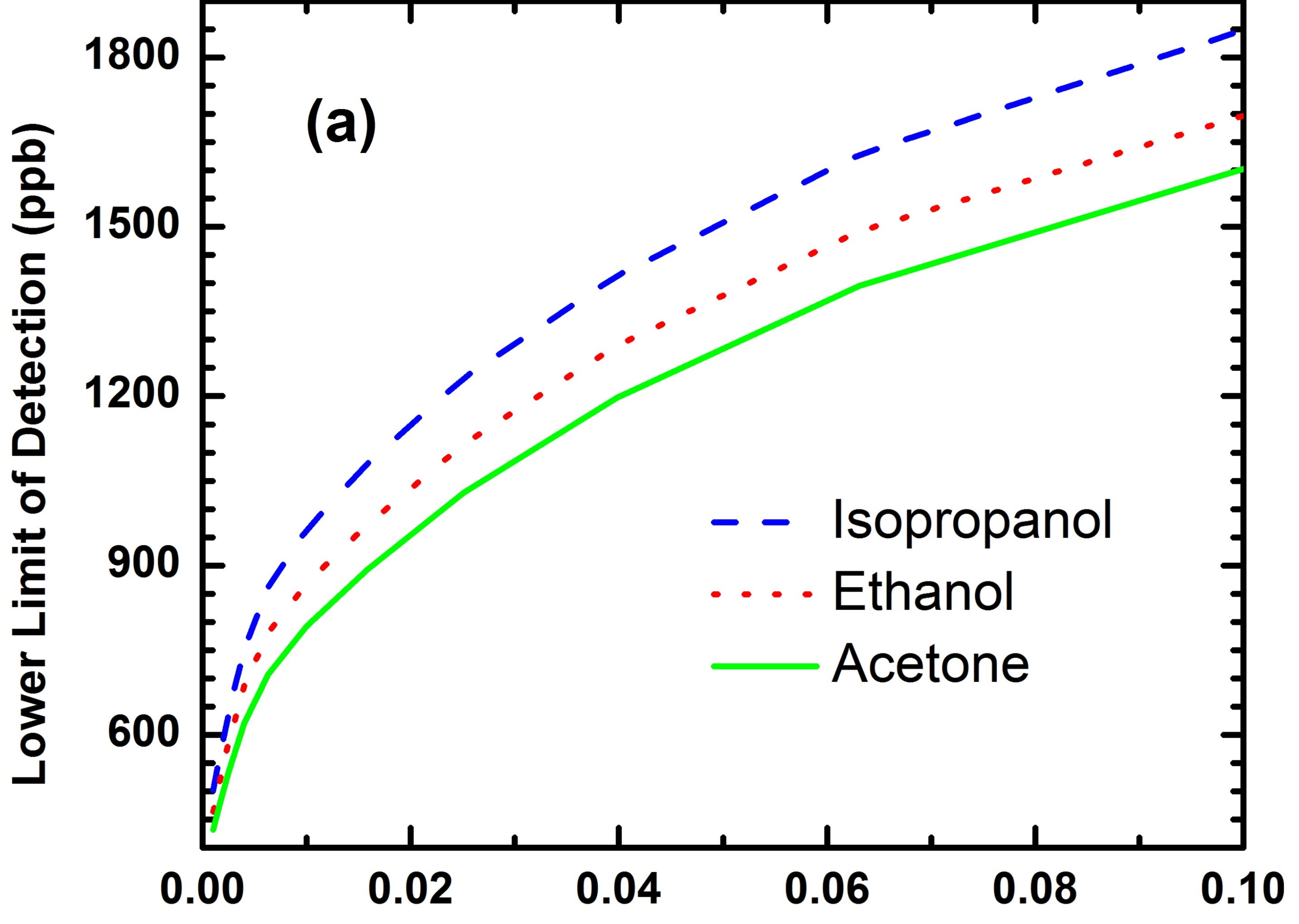

(b) Theoretical Detection Limit of Analytes for $0.001 \%$ Change in Current at Threshold Voltage

\begin{tabular}{|c||c|}
\hline Analyte & Detection Limit (ppb) \\
\hline Isopropanol & 502.42 \\
\hline Ethanol & 465.31 \\
\hline Methanol & 455.93 \\
\hline Toluene & 448.51 \\
\hline \hline Hexane & 441.65 \\
\hline Acetone & 432.72 \\
\hline \hline Diethyl Ether & 408.33 \\
\hline
\end{tabular}

Change in Current at Threshold Voltage, $\Delta \mathrm{l} / \mathrm{I}_{0}(\%)$ 